\title{
A folk theorem for bargaining games
}

Citation for published version (APA):

Herings, P. J. J., Meshalkin, A. V., \& Predtetchinski, A. (2012). A folk theorem for bargaining games.

METEOR, Maastricht University School of Business and Economics. METEOR Research Memorandum No. 055 https://doi.org/10.26481/umamet.2012055

Document status and date:

Published: 01/01/2012

DOI:

10.26481/umamet.2012055

Document Version:

Publisher's PDF, also known as Version of record

\section{Please check the document version of this publication:}

- A submitted manuscript is the version of the article upon submission and before peer-review. There can be important differences between the submitted version and the official published version of record.

People interested in the research are advised to contact the author for the final version of the publication, or visit the DOI to the publisher's website.

- The final author version and the galley proof are versions of the publication after peer review.

- The final published version features the final layout of the paper including the volume, issue and page numbers.

Link to publication

\footnotetext{
General rights rights.

- You may freely distribute the URL identifying the publication in the public portal. please follow below link for the End User Agreement:

www.umlib.nl/taverne-license

Take down policy

If you believe that this document breaches copyright please contact us at:

repository@maastrichtuniversity.nl

providing details and we will investigate your claim.
}

Copyright and moral rights for the publications made accessible in the public portal are retained by the authors and/or other copyright owners and it is a condition of accessing publications that users recognise and abide by the legal requirements associated with these

- Users may download and print one copy of any publication from the public portal for the purpose of private study or research.

- You may not further distribute the material or use it for any profit-making activity or commercial gain

If the publication is distributed under the terms of Article $25 \mathrm{fa}$ of the Dutch Copyright Act, indicated by the "Taverne" license above, 


\section{Maastricht University}

P.J.J. Herings, A. Meshalkin,

A. Predtetchinski

A Folk Theorem for Bargaining Games

$\mathrm{RM} / 12 / 055$

\section{METEOR}

Maastricht University School of Business and Economics

Maastricht Research School of Economics

of Technology and Organization

PO. Box 616

NL-6200 MD Maastricht

The Netherlands 


\title{
A Folk Theorem for Bargaining Games
}

\author{
P. Jean-Jacques Herings*, Andrey Meshalkin $†$ Arkadi Predtetchinski ${ }^{\ddagger}$
}

November 20, 2012

\begin{abstract}
We study strategies with one-period recall in the context of a general class of multilateral bargaining games. A strategy has one-period recall if actions in a particular period are only conditioned on information in the previous and the current period. We show that if players are sufficiently patient, given any proposal in the space of possible agreements, there exists a subgame perfect equilibrium such that the given proposal is made and unanimously accepted in period zero. Our strategies are pure and have one-period recall, and we do not make use of a public randomization device. The players' discount factors are allowed to be heterogeneous.
\end{abstract}

KEYwORDs: dynamic games, bargaining, folk theorem, subgame perfect equilibrium, oneperiod recall.

JEL CODES: C72, C78

*P.J.J. Herings (P.Herings@maastrichtuniversity.nl). Department of Economics, Maastricht University. $\dagger$ A. Meshalkin (A.Rybakov@maastrichtuniversity.nl), Department of Economics, Maastricht University.

${ }^{\ddagger}$ A. Predtetchinski (A.Predtetchinski@maastrichtuniversity.nl), Department of Economics, Maastricht University. 


\section{Introduction}

One of the most important problems in economic theory is the bargaining problem. The bargaining problem studies how agents make an agreement when they can achieve a particular set of feasible payoffs by collaborating. A bargaining game consists of a sequence of proposals and responses to the proposals. If a proposal is accepted by all the players, the game ends. If a proposal is rejected by at least one player, the game continues and the next proposal is made.

In this paper we prove a folk theorem for bargaining games. Folk theorems constitute a class of theorems which state that any individually rational outcome can be sustained as an equilibrium. Early contributions to the folk theorem literature are by Friedman (1971) and by Rubinstein (1979). Fudenberg and Maskin (1986) have proved a folk theorem in repeated games with discounting, where subgame perfect equilibrium is used as the solution concept. Since bargaining games do not belong to the class of repeated games, they are not covered by these results.

Fudenberg and Yamamoto (2011) prove a folk theorem for stochastic games generalizing an earlier result by Dutta (1995). A crucial assumption in both contributions is that of irreducibility: starting from any given state, any other state is visited with a positive probability, irrespectively of the moves of any particular player. In bargaining games, some of the states are terminal, and the irreducibility condition is clearly violated. Moreover both Dutta (1995) and Fudenberg and Yamamoto (2011) assume the set of states and the set of actions to be finite, whereas a large part of the bargaining literature studies infinite action sets. The existing folk theorems for repeated games and for stochastic games therefore do not cover the bargaining model.

One of the main results in the field of bargaining has been proved in Rubinstein (1982). Rubinstein (1982) studies two-person alternating offers bargaining and shows that there is a unique subgame perfect equilibrium in this model. In the unique subgame perfect equilibrium, the proposal of the first proposer is immediately accepted by his opponent. The folk theorem does evidently not hold for two-player bargaining games. We therefore study bargaining games with at least three players in this paper.

The proof of Rubinstein (1982) does not work for bargaining problems with more than two players. As reported in Binmore, Osborne, and Rubinstein (1992), one of the first extensions to the three-person case was made by Shaked. In Shaked's example, Player 1 starts by making a proposal which describes each player's share of a unit surplus. The other players must accept or reject this proposal sequentially. If the proposal is accepted by all players, it is implemented and the game ends. If the proposal is rejected by one of the players, next period begins and Player 2 makes a new proposal. Negotiation continues 
in this way. It is shown that any efficient payoff vector can be supported by subgame perfect equilibrium strategies if the common discount factor is sufficiently high.

Herrero (1985) obtains a result similar to Shaked for the case with three or more players, though we will explain that the construction used in Herrero (1985) is not complete. Haller (1986) also considers the case with three or more players in a game where players vote simultaneously on a proposal. Haller (1986) shows that any efficient division of a unit surplus can be supported as a subgame perfect equilibrium if the common discount factor is sufficiently high.

All constructions used in the literature so far rely on strategies which require infinite recall for all players. The action of a player in a given time-period depends on the whole history of play. In particular, the strategy of every player at any given time-period depends on the actual play in period zero. Infinite recall allows for the punishment of a player, who has deviated from his strategy only once, during the whole remainder of the game. Several authors have questioned the plausibility of such behavior.

Aumann (1981) discusses some of the options to narrow down the definition of equilibrium to avoid unreasonable predictions and mentions bounded recall as a way of modeling bounded rationality in repeated games. Sabourian (1998) characterizes the set of bounded recall pure subgame perfect equilibria in a setting without discounting. His results indicate that the equilibrium set expends fast in the length of recall. Cole and Kocherlakota (2005) show in a context with imperfect public monitoring that for some parameter settings the assumption of bounded recall may reduce the set of equilibrium payoffs to a singleton. To obtain such a result, however, they also make strong symmetry assumptions with respect to the strategies under consideration. Bhaskar, Mailath, and Morris (2009) study subgame perfect equilibria that are purifiable and have bounded recall. Equilibrium strategies are purifiable if they also constitute an equilibrium of a perturbed game with independent private payoff perturbations in the sense of Harsanyi (1973). They show that only Markovian equilibria have bounded recall and are purifiable.

We consider a general specification of the multilateral bargaining model and explore the existence of subgame perfect equilibria under the strong bounded recall restriction that players' actions may only be conditional on actions in the previous and the current period. We make only weak assumptions on the set of feasible payoffs.

Our bargaining protocol is sufficiently general to cover many existing models. Special cases of our bargaining protocol with alternating or rotating proposers are described in Rubinstein (1982) and Herrero (1985). We cover protocols with time-invariant recognition probabilities as studied in Binmore (1987) and Banks and Duggan (2000). Models where the proposer is selected by means of an underlying Markov process generalize these ap- 
proaches, see Merlo and Wilson (1995), Kalandrakis (2006), and Herings and Predtetchinski (2010), and are also special cases of our bargaining protocol. Part of the literature studies endogenous bargaining protocols, where a player who rejects becomes the next proposer, a bargaining protocol introduced in Selten (1981) and also used in Chatterjee, Dutta, Ray, and Sengupta (1993) in coalitional bargaining theory. Our set-up allows for endogenous protocols as well, where not only rejections affect the choice of the next proposer, but also the contents of previous proposals may influence this choice. Our bargaining protocol is allowed to have infinite recall.

We prove a folk theorem for this general class of multilateral bargaining games. Given any payoff vector in the set of feasible payoffs, we construct a strategy profile with oneperiod recall where the given payoff vector is proposed and accepted in period zero. The strategy profile is shown to be a subgame perfect equilibrium when all players' discount factors are above a particular threshold. The constructed strategy profile is pure and we do not rely on a public randomization device to establish our main result.

We allow discount factors to be heterogeneous, a case that is hardly studied in the folk theorem literature. Unlike the classical folk theorem in Fudenberg and Maskin (1986), Lehrer and Pauzner (1999) find that in two-player repeated games with heterogeneous discount factors, not all feasible individually rational payoffs can be supported by an equilibrium, even when both players become very patient. They also point out that it is difficult to characterize the feasible payoff set in an $n$-player repeated game. Chen (2008) shows the possibility of a player obtaining a subgame perfect equilibrium payoff below his effective minimax value in a three-player repeated game. As our main result demonstrates, no such difficulties arise for multilateral bargaining games.

The rest of the paper is organized as follows. In Section 2 we discuss our class of multilateral bargaining games and we describe how existing models fit into it. In Sections 3 and 4 we analyze the example of Shaked and the results of Herrero (1985). We show that these results rely on the use of strategies with infinite recall. In Section 5 we present a motivating example, which illustrates how a payoff vector can be supported as a subgame perfect equilibrium in one-period recall strategies. In Section 6 we prove the main result, the folk theorem for a general class of multilateral bargaining games, and Section 7 concludes.

\section{Bargaining games}

We consider a dynamic game of perfect information $\Gamma$, where a set of $N$ players with cardinality $n \geq 3$ has to agree on the choice of a payoff vector in a set of feasible payoffs $V$. We use the notation $e_{0}$ for the vector $(0, \ldots, 0)$, and $e_{i}$ for the $i$-th unit vector in $\mathbb{R}^{N}$. 
We make four assumptions on the set of feasible payoffs $V$ :

1. $V$ is a closed subset of $\mathbb{R}_{+}^{N}$.

2. $\forall v \in V, \forall i \in N, v_{i} \leq 1$.

3. $e_{0} \in V, \forall i \in N, e_{i} \in V$.

4. $\forall v \in V$, the set $\left\{i \in N \mid v_{i}=1\right\}$ contains at most one point.

These four assumptions are satisfied in most applications and are not very restrictive. Closedness is a standard technical assumption. The second assumption is just a normalization of the set $V$. Any bounded set of feasible payoffs satisfies this assumption after an appropriate linear transformation of the payoffs. The first and second assumption together plus the standard assumption that the bargaining problem is essential implies that we can assume without loss of generality that for every $i \in N$ there is some $v \in V$ such that $v_{i}=1$. Together with the standard requirement that $V$ is comprehensive from below, this would imply the third assumption. The last assumption says that it is not possible for two players to reach their maximum utility level simultaneously. This assumption would be satisfied if players can make some transfers to each other. Notice that our assumptions permit the set $V$ to be non-convex or to consist of a discrete number of points.

In each time period $t=0,1,2, \ldots$ nature selects the proposer and the order of responders by means of a permutation $\pi^{t}:\{1, \ldots, n\} \rightarrow N$. The numbers $\{1, \ldots, n\}$ are the positions of the players in the bargaining protocol. Player $\pi^{t}(1)$ makes a proposal $x^{t} \in V$, after which Player $\pi^{t}(2)$ responds to the proposal by accepting or rejecting it. If Player $\pi^{t}(2)$ accepts, it is Player $\pi^{t}(3)$ 's turn to respond to the proposal and so on. If all players accept the proposal, the game ends and the proposal is implemented. If one of the players rejects the proposal, the next period begins. In the next time period nature again selects a permutation, which determines the proposer and the order of the players to respond to the proposal. The utility of Player $i \in N$ who receives outcome $x_{i}$ in period $t$ is $\delta_{i}^{t} x_{i}$, where $\delta_{i} \in[0,1)$ is the discount factor for Player $i$. The utility of perpetual disagreement is 0 for all players.

Our bargaining protocol is very general. There are no restrictions on the process by which the permutation is selected in each round. In particular, in any round the probability distribution over the set of all permutations might be dependent on the entire history of play. Special cases of the protocol are described in Selten (1981), Herrero (1985), Binmore (1987), Chatterjee et al. (1993), Merlo and Wilson (1995), Banks and Duggan (2000), Kalandrakis (2006), and Herings and Predtetchinski (2010).

In Selten (1981) and Chatterjee et al. (1993) there is an endogenous protocol for the 
players: the player who rejects is the next proposer. In this case the permutation in each period depends on the history of play in the previous period. Herrero (1985) considers a model with players that rotate in making offers. In our model, this is achieved by specifying $\pi^{t}(k)=t+k \bmod n$. In Binmore (1987) and Banks and Duggan (2000) the proposer selection process is modeled by time-invariant recognition probabilities. The probability that Player $i \in N$ is recognized to make a proposal is constant across all periods. In Merlo and Wilson (1995), Kalandrakis (2006), and Herings and Predtetchinski (2010), the order in which players act in any period is determined by a Markov process: if the state of the game is $s$, then the game moves to state $s^{\prime}$ with probability $p\left(s, s^{\prime}\right)$. In other words, the current state depends on the previous state and actions of the players do not influence the state, a specification that is allowed for in our model. Our bargaining protocol even allows for the next proposer to depend on the current proposal, or on proposals made in the past, something which is not covered in the bargaining literature so far.

A history $h$ is a sequence of actions that have occurred before a particular decision node in the game. There are three different types of histories. Every period there are histories ending with a move by nature, there are histories ending with a move by the proposer, and there are histories ending with a move by a responder. Any non-terminal history is of one of the following three types:

1. $h \in H_{1}^{t}$ if and only if $h=\left(\pi^{0}, x^{0}, r^{0}, \ldots, \pi^{t-1}, x^{t-1}, r^{t-1}, \pi^{t}\right)$,

2. $h \in H_{2}^{t}$ if and only if $h=\left(\pi^{0}, x^{0}, r^{0}, \ldots, \pi^{t-1}, x^{t-1}, r^{t-1}, \pi^{t}, x^{t}\right)$,

3. $h \in H_{3}^{t}$ if and only if $h=\left(\pi^{0}, x^{0}, r^{0}, \ldots, \pi^{t-1}, x^{t-1}, r^{t-1}, \pi^{t}, x^{t}, r^{t}\right)$,

where $\pi^{k}$ is a permutation of the set $N, x^{k} \in V$, and $r^{k} \in N \backslash\left\{\pi^{k}(1)\right\}$ for every $k$. After a history $h \in H_{1}^{t}$ the proposer moves, after a history $h \in H_{2}^{t}$ a responder moves, and after a history $h \in H_{3}^{t}$ nature moves. In principle, one has to distinguish between histories after which the first responder moves, histories after which the second responder moves, and so on. We shall not make such a distinction; the symbol $h \in H_{2}^{t}$ might denote any of these histories. When Player $\pi^{t}(j)$ casts a vote after history $h$, it is to be understood that the Players $\pi^{t}(2), \ldots, \pi^{t}(j-1)$ have already accepted the proposal. Histories in $H_{1}^{t}$ are called proposer histories and those in $H_{2}^{t}$ responder histories. We denote the union of $H_{1}^{t}, H_{2}^{t}$, and $H_{3}^{t}$ over $t=0,1, \ldots$ by $H_{1}, H_{2}$, and $H_{3}$, respectively.

A strategy $\sigma_{i}$ of Player $i$ is a function that assigns a point in $V$ to each proposer history $h \in H_{1}$ ending with a permutation $\pi^{t}$ such that $\pi^{t}(1)=i$, and an element in \{accept, reject to each responder history $h \in H_{2}$ with the last permutation $\pi^{t}$ being such that $\pi^{t}(1) \neq i$.

A strategy profile is denoted by $\sigma=\left(\sigma_{i}\right)_{i \in N}$. Given a strategy profile $\sigma$ and a history $h \in H_{1}^{t}$, we use $p^{t}(h)$ for the resulting proposal. 
A strategy profile $\sigma$ is said to have the immediate acceptance property if for every $t \geq 0$ and every history $h \in H_{1}^{t}$, the proposal $p^{t}(h)$ is accepted by all responders. If a strategy profile $\sigma$ has the immediate acceptance property, then the first proposal to be made is accepted, and the game ends in period 0 .

A strategy $\sigma_{i}$ has $K$-recall if it assigns the same action to Player $i$ for all histories of the same length that coincide in the last $K$ periods. More precisely, a strategy $\sigma_{i}$ has $K$-recall if for all histories $h$ and $\bar{h}$ after which Player $i$ moves, it holds that $\sigma_{i}(h)=\sigma_{i}(\bar{h})$ whenever one of the following cases is true for some $t=0,1, \ldots$ :

1. $h, \bar{h} \in H_{1}^{t}$, for $k=t-K, \ldots, t-1: \pi^{k}=\bar{\pi}^{k}, x^{k}=\bar{x}^{k}, r^{k}=\bar{r}^{k}$, and $\pi^{t}=\bar{\pi}^{t}$;

2. $h, \bar{h} \in H_{2}^{t}$, for $k=t-K, \ldots, t-1: \pi^{k}=\bar{\pi}^{k}, x^{k}=\bar{x}^{k}, r^{k}=\bar{r}^{k}$, and $\pi^{t}=\bar{\pi}^{t}, x^{t}=\bar{x}^{t}$.

A strategy $\sigma_{i}$ has infinite recall if it does not have $K$-recall for any $K$.

A strategy profile $\sigma$ has $K$-recall if every strategy $\sigma_{i}, i \in N$, has $K$-recall. A strategy profile $\sigma$ has infinite recall if it does not have $K$-recall for any $K$.

In this paper we use the concept of subgame perfect equilibrium (SPE) as the solution concept. A strategy profile is a subgame perfect equilibrium if it induces a Nash equilibrium in every subgame of the original game.

To show that a strategy profile $\sigma$ is a subgame perfect equilibrium we show that there are no profitable one-shot deviations from $\sigma$ at any history of the game. Consider a nonterminal history $h$ after which Player $i$ moves. A one-shot deviation by Player $i$ at history $h$ is a strategy $\sigma_{i}^{\prime}$ for Player $i$ that agrees with $\sigma_{i}$ on every non-terminal history except $h$. It is said to be profitable if, conditional on the history $h$ being reached, the payoff to Player $i$ from $\left(\sigma_{i}^{\prime}, \sigma_{-i}\right)$ is higher than that from $\sigma$.

As our bargaining game is an infinite horizon discounted multi-stage game with observed actions that is continuous at infinity, the following theorem applies.

Theorem 2.1 Fudenberg and Tirole (1991). The strategy profile $\sigma$ is a subgame perfect equilibrium of $\Gamma$ if and only if no player has a profitable one-shot deviation from $\sigma$ at any history.

The bargaining literature uses either sequential or simultaneous voting. For instance, Herrero (1985) uses sequential voting, whereas Haller (1986) considers simultaneous voting. Sequential voting leads to more subgames than simultaneous voting, thereby increasing the power of subgame perfection. A folk theorem for bargaining games derived under sequential voting is therefore a stronger result. 


\section{Shaked's example}

In this section we revisit an example of a 3-player divide-the-dollar bargaining game from Binmore, Osborne, and Rubinstein (1992, pp. 191-192), where the example is attributed to Shaked. It is argued that, for any efficient division of the dollar, there exists a subgame perfect equilibrium such that the given division is proposed and accepted in period 0 . We extend the subgame perfect equilibrium strategies constructed in Binmore, Osborne, and Rubinstein (1992) to also include inefficient divisions of the dollar and show that they have infinite recall.

Example 3.1 Consider a game with $N=\{1,2,3\}$ and $V=\left\{x \in \mathbb{R}_{+}^{3} \mid x_{1}+x_{2}+x_{3} \leq 1\right\}$. The players have a common discount factor $\delta$. The bargaining game proceeds as described in Section 2, where the bargaining protocol is defined in Table 1.

\begin{tabular}{|c|c|c|c|c|c|}
\hline & $t=0$ & $t=1$ & $t=2$ & $t=3$ & $\ldots$ \\
\hline$\pi^{t}(1)$ & 1 & 2 & 3 & 1 & $\ldots$ \\
\hline$\pi^{t}(2)$ & 2 & 3 & 1 & 2 & $\ldots$ \\
\hline$\pi^{t}(3)$ & 3 & 1 & 2 & 3 & $\ldots$ \\
\hline
\end{tabular}

Table 1: The bargaining protocol in Example 3.1.

Theorem 3.2 Consider the bargaining game of Example 3.1. If $1 / 2<\delta<1$, then for every proposal $a \in V$, there exists an SPE in which at $t=0$ the proposal a is made by Player 1 and accepted by Players 2 and 3.

We now define a strategy profile which satisfies the requirements of Theorem 3.2. For each $x \in V$ and each $t \geq 0$, we define

$$
i^{t+1}(x)=\min \left\{k \neq \pi^{t}(1) \mid x_{k} \leq 1 / 2\right\} .
$$

We inductively define the sequence $s^{0}, s^{1}, \ldots$ of functions, where $s^{t}: V^{t} \rightarrow V$ with $V^{t}$ the $t$-fold product of $V$. We define $s^{0}=a$ and

$$
s^{t+1}\left(x^{0}, \ldots, x^{t}\right)= \begin{cases}s^{t}\left(x^{0}, \ldots, x^{t-1}\right), & \text { if } x_{\pi^{t}(1)}^{t} \leq s_{\pi^{t}(1)}^{t}\left(x^{0}, \ldots, x^{t-1}\right), \\ e_{i^{t+1}\left(x^{t}\right)}, & \text { if } x_{\pi^{t}(1)}^{t}>s_{\pi^{t}(1)}^{t}\left(x^{0}, \ldots, x^{t-1}\right) .\end{cases}
$$

The function $s^{t}$ represents the "state of mind" of the players in period $t$ and corresponds to the proposal that should be made in period $t$.

We define the strategy profile $\sigma$ as follows: 
1. For each $t \geq 0$, for each history $h=\left(\pi^{0}, x^{0}, r^{0}, \ldots, \pi^{t-1}, x^{t-1}, r^{t-1}, \pi^{t}\right)$, Player $\pi^{t}(1)$ proposes $p^{t}(h)=s^{t}\left(x^{0}, \ldots, x^{t-1}\right)$.

2. The player who responds first after history $\left(\pi^{0}, x^{0}, r^{0}, \ldots, \pi^{t}, x^{t}\right)$, i.e. $\pi^{t}(2)$, accepts $x^{t}$ if $x_{\pi^{t}(2)}^{t} \geq \delta s_{\pi^{t}(2)}^{t+1}\left(x^{0}, \ldots, x^{t}\right)$ and rejects otherwise.

3. The player who responds second after history $\left(\pi^{0}, x^{0}, r^{0}, \ldots, \pi^{t}, x^{t}\right)$, i.e. $\pi^{t}(3)$, accepts $x^{t}$ if $x_{\pi^{t}(3)}^{t} \geq \delta s_{\pi^{t}(3)}^{t+1}\left(x^{0}, \ldots, x^{t}\right)$ and rejects otherwise.

Claim 3.3 The strategy $\sigma$ has the immediate acceptance property. In particular, according to $\sigma$, Player 1 proposes a in period 0, which is accepted by both Players 2 and 3.

Proof. Consider a proposer history $h=\left(\pi^{0}, x^{0}, r^{0}, \ldots, \pi^{t-1}, x^{t-1}, r^{t-1}, \pi^{t}\right)$. We have to show that the proposal $p^{t}(h)$ is accepted by both responders. Indeed, if Player $\pi^{t}(1)$ makes the proposal $x^{t}=p^{t}(h)=s^{t}\left(x^{0}, \ldots, x^{t-1}\right)$, then $s^{t+1}\left(x^{0}, \ldots, x^{t}\right)=s^{t}\left(x^{0}, \ldots, x^{t-1}\right)=x^{t}$ and so $x_{i}^{t} \geq \delta s_{i}^{t+1}\left(x^{0}, \ldots, x^{t}\right)$ for every $i$. Hence both responders accept $x^{t}$ as desired. The second part of the claim follows since $p^{0}\left(\pi^{0}\right)=a$.

Claim 3.4 If $1 / 2<\delta<1$, then $\sigma$ is an SPE.

Proof. We show that at every history none of the players has a profitable one-shot deviation from $\sigma$.

SteP 1. Consider a proposer history $\left(\pi^{0}, x^{0}, r^{0}, \ldots, \pi^{t-1}, x^{t-1}, r^{t-1}, \pi^{t}\right)$. According to the strategy $\sigma$ Player $\pi^{t}(1)$ makes the proposal $s^{t}\left(x^{0}, \ldots, x^{t-1}\right)$, which is accepted by both responders according to Claim 3.3. Thus $\sigma$ results in a payoff of $\delta^{t} s_{\pi^{t}(1)}^{t}\left(x^{0}, \ldots, x^{t-1}\right)$ to Player $\pi^{t}(1)$.

Consider a one-shot deviation by Player $\pi^{t}(1)$, who proposes a point $x^{t}$ such that $x_{\pi^{t}(1)}^{t} \leq s_{\pi^{t}(1)}^{t}\left(x^{0}, \ldots, x^{t-1}\right)$. It follows that $s^{t+1}\left(x^{0}, \ldots, x^{t}\right)=s^{t}\left(x^{0}, \ldots, x^{t-1}\right)$. Player $\pi^{t}(1)$ then receives the payoff $\delta^{t} x_{\pi^{t}(1)}^{t} \leq \delta^{t} s_{\pi^{t}(1)}^{t}\left(x^{0}, \ldots, x^{t-1}\right)$ if $x^{t}$ is accepted by both responders, and $\delta^{t+1} s_{\pi^{t}(1)}^{t+1}\left(x^{0}, \ldots, x^{t}\right)=\delta^{t} s_{\pi^{t}(1)}^{t}\left(x^{0}, \ldots, x^{t-1}\right)$ if $x^{t}$ is rejected. The one-shot deviation is not profitable.

Consider a one-shot deviation by Player $\pi^{t}(1)$, who proposes a point $x^{t}$ such that $x_{\pi^{t}(1)}^{t}>s_{\pi^{t}(1)}^{t}\left(x^{0}, \ldots, x^{t-1}\right)$. We then have $s^{t+1}\left(x^{0}, \ldots, x^{t}\right)=e_{i}$, where $i=\min \left\{k \neq \pi^{t}(1)\right.$ $\left.x_{k}^{t} \leq 1 / 2\right\}$. According to the strategy profile $\sigma, x^{t}$ would be rejected by Player $i$, because Player $i$ is the only player with $x_{i}^{t}<\delta s_{i}^{t+1}\left(x^{0}, \ldots, x^{t}\right)$. Hence in period $t+1$ the proposal $e_{i}$ will be made and accepted, resulting in payoff 0 for Player $\pi^{t}(1)$.

We conclude that there are no profitable one-shot deviations at the proposer history $h$.

SteP 2. Consider a responder history $h=\left(\pi^{0}, x^{0}, r^{0}, \ldots, \pi^{t}, x^{t}\right)$. We check that Player $\pi^{t}(2)$ has no profitable one-shot deviation after $h$. 
Suppose $x^{t}$ is such that $x_{\pi^{t}(2)}^{t} \geq \delta s_{\pi^{t}(2)}^{t+1}\left(x^{0}, \ldots, x^{t}\right)$, so $x^{t}$ should be accepted according to $\sigma$, leading to a payoff of $\delta^{t} x_{\pi^{t}(2)}^{t}$ if Player $\pi^{t}(3)$ also accepts $x^{t}$, or $\delta^{t+1} s_{\pi^{t}(2)}^{t+1}\left(x^{0}, \ldots, x^{t}\right)$ if Player $\pi^{t}(3)$ rejects $x^{t}$. In either case the payoff to Player $\pi^{t}(2)$ is at least $\delta^{t+1} s_{\pi^{t}(2)}^{t+1}\left(x^{0}, \ldots, x^{t}\right)$. If Player $\pi^{t}(2)$ deviates and rejects $x^{t}$, next period the proposal $s^{t+1}\left(x^{0}, \ldots, x^{t}\right)$ will be made and accepted. Then Player $\pi^{t}(2)$ receives payoff $\delta^{t+1} s_{\pi^{t}(2)}^{t+1}\left(x^{0}, \ldots, x^{t}\right)$. We conclude that rejection is not a profitable deviation.

Suppose $x^{t}$ is such that $x_{\pi^{t}(2)}^{t}<\delta s_{\pi^{t}(2)}^{t+1}\left(x^{0}, \ldots, x^{t}\right)$, so $\sigma$ prescribes rejection, leading to a payoff of $\delta^{t+1} s_{\pi^{t}(2)}^{t+1}\left(x^{0}, \ldots, x^{t}\right)$. Suppose Player $\pi^{t}(2)$ deviates and accepts $x^{t}$. He then receives payoff $\delta^{t} x_{\pi^{t}(2)}^{t}$ if Player $\pi^{t}(3)$ also accepts $x^{t}$, or $\delta^{t+1} s_{\pi^{t}(2)}^{t+1}\left(x^{0}, \ldots, x^{t}\right)$ if Player $\pi^{t}(3)$ rejects $x^{t}$. In either case acceptance results in a payoff of at most $\delta^{t+1} s_{\pi^{t}(2)}^{t+1}\left(x^{0}, \ldots, x^{t}\right)$. We conclude that acceptance is not a profitable deviation. We have checked that there is no profitable one-shot deviation for Player $\pi^{t}(2)$.

SteP 3. We check that Player $\pi^{t}(3)$ has no profitable one-shot deviation at the history $h=\left(\pi^{0}, x^{0}, r^{0}, \ldots, \pi^{t}, x^{t}\right)$.

Suppose $x^{t}$ is such that $x_{\pi^{t}(3)}^{t} \geq \delta s_{\pi^{t}(3)}^{t+1}\left(x^{0}, \ldots, x^{t}\right)$, so strategy $\sigma$ prescribes acceptance and leads to payoff $\delta^{t} x_{\pi^{t}(3)}^{t}$. If $\pi^{t}(3)$ deviates and rejects $x^{t}$, he only receives $\delta^{t+1} s_{\pi^{t}(3)}^{t+1}\left(x^{0}, \ldots, x^{t}\right)$. We conclude that rejection is not a profitable deviation.

Suppose $x^{t}$ is such that $x_{\pi^{t}(3)}^{t}<\delta s_{\pi^{t}(3)}^{t+1}\left(x^{0}, \ldots, x^{t}\right)$. The strategy $\sigma$ prescribes rejection and leads to payoff $\delta^{t+1} s_{\pi^{t}(3)}^{t+1}\left(x^{0}, \ldots, x^{t}\right)$. If $\pi^{t}(3)$ accepts $x^{t}$, he only receives $\delta^{t} x_{\pi^{t}(3)}^{t}$. We conclude that acceptance is not a profitable deviation.

We conclude our discussion of Shaked's construction by showing that the strategy profile $\sigma$ has infinite recall. Notice that the functions $s^{t}$ defining the state of mind of the players are being determined recursively, that is the new state of mind $s^{t+1}$ is computed as a function of the current state $s^{t}$ and the current proposal $x^{t}$. We will now provide sufficient conditions for recursively defined functions to have infinite recall.

Let $X$ and $Y$ be sets with $Y \subset X$. For each $t \geq 1$, let $s^{t}: X^{t} \rightarrow Y$ be a function, where $X^{t}$ is the $t$-fold product of the set $X$. We write $s=\left(s^{0}, s^{1}, \ldots\right)$. The sequence $s$ is said to have 0 -recall if each $s^{t}$ is a constant function. It is said to have $k$-recall for $k \geq 1$ if $s^{t}\left(x^{0}, \ldots, x^{t-1}\right)=s^{t}\left(\bar{x}^{0}, \ldots, \bar{x}^{t-1}\right)$ whenever $t \geq k$ and $x^{\ell}=\bar{x}^{\ell}$ for each $\ell \in\{t-k, \ldots, t-1\}$. The sequence is said to have infinite recall if it does not have $k$-recall for any $k \geq 0$.

Let $a$ be a point of $Y$, and for each $t \geq 1$ let $f^{t}: X \times Y \rightarrow Y$. We define the sequence $s=\left(s^{0}, s^{1}, \ldots\right)$ of functions $s^{t}: X^{t} \rightarrow Y$ by induction on $t$ as follows:

$$
\begin{aligned}
& s^{0}=a, \\
& s^{t+1}\left(x^{0}, \ldots, x^{t}\right)=f^{t+1}\left(x^{t}, s^{t}\left(x^{0}, \ldots, x^{t-1}\right)\right), \quad t \geq 0 .
\end{aligned}
$$


Claim 3.5 Let $f=\left(f^{1}, f^{2}, \ldots\right)$ and $a \in Y$ be such that $[1] f^{t}(a, a)=a$ for each $t \geq 1$, [2] there is a $t \geq 1$ and an $x \in X$ such that $f^{t}(x, a) \neq a$, and [3] for each $t \geq 1$, for every $y, y^{\prime} \in Y$ such that $y \neq y^{\prime}$, there is $x \in X$ such that $f^{t}(x, y) \neq f^{t}\left(x, y^{\prime}\right)$. Then $s$ as defined by (3.2)-(3.3) has infinite recall.

Proof. The proof is by induction on $k$. We show that $s$ does not have 0 -recall. Suppose on the contrary that $s^{t}$ is a constant for each $t$. Then by [1] each $s^{t}$ is identically equal to $a$ on $X^{t}$. But for $t$ and $x$ as in Condition [2] we have that

$$
s^{t}(a, \ldots, a, x)=f^{t}\left(x, s^{t-1}(a, \ldots, a)\right)=f^{t}(x, a) \neq a,
$$

leading to a contradiction.

Assume $s$ does not have $k$-recall for some $k \geq 0$. Then there is $t \geq k$ and there are points $\left(x^{0}, \ldots, x^{t-1}\right),\left(\bar{x}^{0}, \ldots, \bar{x}^{t-1}\right) \in X^{t}$ such that $x^{\ell}=\bar{x}^{\ell}$ for each $\ell \in\{t-k, \ldots, t-1\}$, and

$$
s^{t}\left(x^{0}, \ldots, x^{t-1}\right) \neq s^{t}\left(\bar{x}^{0}, \ldots, \bar{x}^{t-1}\right) .
$$

It then follows by [3] that there is an $x^{t} \in X$ such that $f^{t+1}\left(x^{t}, s^{t}\left(x^{0}, \ldots, x^{t-1}\right)\right) \neq$ $f^{t+1}\left(x^{t}, s^{t}\left(\bar{x}^{0}, \ldots, \bar{x}^{t-1}\right)\right)$, so

$$
s^{t+1}\left(x^{0}, \ldots, x^{t-1}, x^{t}\right) \neq s^{t+1}\left(\bar{x}^{0}, \ldots, \bar{x}^{t-1}, x^{t}\right),
$$

proving that $s$ does not have $k+1-$ recall.

Claim 3.6 The strategy profile $\sigma$ has infinite recall.

Proof. We prove the claim by showing that the sequence $s=\left(s^{0}, s^{1}, \ldots\right)$ of states of mind as defined by Equation (3.1) has infinite recall. We take $X=V$ and $Y=\left\{e_{1}, e_{2}, e_{3}, a\right\}$ and, for $t=1,2,3$, we define the function $f^{t}: X \times Y \rightarrow Y$ by

$$
f^{t}(x, y)= \begin{cases}y, & \text { if } x_{t} \leq y_{t} \\ e_{i^{t}(x)}, & \text { if } x_{t}>y_{t}\end{cases}
$$

where $i^{t}(x)=\min \left\{k \neq t \mid x_{k} \leq 1 / 2\right\}$. For $k \in \mathbb{N}$ and $t=1,2,3$, we define $f^{t+3 k}=f^{t}$. The sequence $s$ as defined in Equation (3.3) is identical to the one defined in (3.1). Table 2) displays some of the values of $i^{t}$ and $f^{t}$.

We verify that $f=\left(f^{1}, f^{2}, \ldots\right)$ meets the conditions of Claim 3.5. By definition of $f^{t}$ we have that $f^{t}(a, a)=a$, so Condition [1] holds. 


\begin{tabular}{|c|ccc|}
\hline$x$ & $i^{1}(x)$ & $i^{2}(x)$ & $i^{3}(x)$ \\
\hline$e_{1}$ & 2 & 3 & 2 \\
$e_{2}$ & 3 & 1 & 1 \\
$e_{3}$ & 2 & 1 & 1 \\
\hline
\end{tabular}

\begin{tabular}{|cc|ccc|}
\hline$x$ & $y$ & $f^{1}(x, y)$ & $f^{2}(x, y)$ & $f^{3}(x, y)$ \\
\hline$e_{1}$ & $e_{1}$ & $e_{1}$ & $e_{1}$ & $e_{1}$ \\
$e_{2}$ & $e_{1}$ & $e_{1}$ & $e_{1}$ & $e_{1}$ \\
$e_{3}$ & $e_{1}$ & $e_{1}$ & $e_{1}$ & $e_{1}$ \\
\hline$e_{1}$ & $e_{2}$ & $e_{2}$ & $e_{2}$ & $e_{2}$ \\
$e_{2}$ & $e_{2}$ & $e_{2}$ & $e_{2}$ & $e_{2}$ \\
$e_{3}$ & $e_{2}$ & $e_{2}$ & $e_{2}$ & $e_{1}$ \\
\hline$e_{1}$ & $e_{3}$ & $e_{2}$ & $e_{3}$ & $e_{3}$ \\
$e_{2}$ & $e_{3}$ & $e_{3}$ & $e_{1}$ & $e_{3}$ \\
$e_{3}$ & $e_{3}$ & $e_{3}$ & $e_{3}$ & $e_{3}$ \\
\hline
\end{tabular}

Table 2: Some of the values of $i^{t}$ and $f^{t}$.

We now verify that Condition [2] holds. If $a$ is not an element of $\left\{e_{1}, e_{2}, e_{3}\right\}$, then we have $f^{1}\left(e_{1}, a\right)=e_{2} \neq a$. If $a=e_{1}$, we take $x=(2 / 3,1 / 3,0)$, and find that $f^{2}\left(x, e_{1}\right)=e_{3} \neq$ $a$. If $a=e_{2}$, we have $f^{3}\left(e_{3}, e_{2}\right)=e_{1} \neq a$. If $a=e_{3}$, we have $f^{1}\left(e_{1}, e_{3}\right)=e_{2} \neq a$.

To verify [3], let $y$ and $y^{\prime}$ be two distinct points of $Y$. If $y$ is an element of $\left\{e_{1}, e_{2}, e_{3}\right\}$ while $y^{\prime}=a$ is not, then $f^{t}(a, a)=a \neq f^{t}\left(e_{t}, a\right)$ since $f^{t}\left(e_{t}, a\right)$ is an element $\left\{e_{1}, e_{2}, e_{3}\right\}$. If both $y$ and $y^{\prime}$ are members of $\left\{e_{1}, e_{2}, e_{3}\right\}$, then the condition follows from Table 2. This completes the proof.

\section{Herrero-Haller's example}

In this section we re-examine the example of an $n$-player divide-the-dollar game as studied in Herrero (1985). Herrero claims that given any division of the dollar, there is a subgame perfect perfect equilibrium such that the given division is proposed and unanimously accepted in period zero. We quote Herrero's definition of the strategy profile and argue that it is incomplete. We next reproduce the proof of the claim given by Hans Haller (private communication). We show that the strategies used in Haller's proof have infinite recall.

Example 4.1 Consider a game with $N=\{1, \ldots, n\}$ and $V=\left\{x \in \mathbb{R}_{+}^{n} \mid x_{1}+\cdots+x_{n} \leq 1\right\}$. The players have a common discount factor $\delta$. The game proceeds as in Section 2, with the sequence of moves given by the following rule:

For the case $n=3$, the game coincides with the one in the preceding section.

Theorem 4.2 Consider the bargaining game of Example 4.1. If $1 /(n-1)<\delta<1$, then 


\begin{tabular}{|c|ccccccc|}
\hline & $t=0$ & $t=1$ & $t=2$ & $\ldots$ & $t=n-1$ & $t=n$ & $\ldots$ \\
\hline$\pi^{t}(1)$ & 1 & 2 & 3 & $\ldots$ & $n$ & 1 & $\ldots$ \\
$\pi^{t}(2)$ & 2 & 3 & 4 & $\ldots$ & 1 & 2 & $\ldots$ \\
$\pi^{t}(3)$ & 3 & 4 & 5 & $\ldots$ & 2 & 3 & $\ldots$ \\
$\vdots$ & $\vdots$ & $\vdots$ & $\vdots$ & $\vdots$ & $\vdots$ & $\vdots$ & $\vdots$ \\
$\pi^{t}(n-1)$ & $n-1$ & $n$ & 1 & $\ldots$ & $n-2$ & $n-1$ & $\ldots$ \\
$\pi^{t}(n)$ & $n$ & 1 & 2 & $\ldots$ & $n-1$ & $n$ & $\ldots$ \\
\hline
\end{tabular}

Table 3: The bargaining protocol in Example 4.1.

for every proposal $a \in V$, there exists an SPE in which at $t=0$ the proposal a is made by Player 1 and accepted by Players 2 and 3.

Herrero (1985) defines the following strategy profile in an attempt to prove Theorem 4.2.

1. Player 1 proposes $a$, which is unanimously accepted at time 0 .

2. $a$ is proposed and accepted at time $t$, provided $a$ was proposed at time $t-1$ (all $t>0)$.

3. If Player $j$ deviates from (1) or (2) and proposes $y$ such that $y_{k}<\delta$ for some $k \neq j$ at time $\tau$, then

(a) Player $k$ rejects $y$

(b) $e_{k}$ is proposed and accepted at time $\tau+1$

(c) $e_{k}$ is proposed and accepted at time $t$, provided $e_{k}$ was proposed at time $t-1$ $($ all $t>\tau+1)$

(d) and, if Player $l$ deviates from (b) or (c) to propose $z$ with $z_{h}<\delta$ for some $h \neq l$ at time $T$, then begin (a) again with $j=l$ and $k=h$.

Unfortunately, the above definition appears to be incomplete: if under item 3 there is more than one Player $k \neq j$ with $y_{k}<\delta$ it is not clear which one should be chosen. To illustrate this difficulty, consider a history where in period 0 Player 1 proposes $y=$ $(\delta,(1-\delta) / 2,(1-\delta) / 2) \neq a$ and Player 2 accepts. It is now Player 3's turn to respond to the proposal. Notice that $y_{2}<\delta$ and $y_{3}<\delta$. Should Player 3 accept or reject the proposal $y$ ? This question cannot be answered unless it is decided whether the next proposal is going to be $e_{2}$ or $e_{3}$ if Player 3 rejects $y$. 
It is clear that one can interpret Conditions 1-3 in a number of ways. One such interpretation is due to Haller (private communication) and is reproduced below. For each $t \geq 0$, for each $x \in V$, we define

$$
i^{t+1}(x)=\min \left\{k \neq \pi^{t}(1) \mid x_{k} \leq \delta\right\} .
$$

This expression is well-defined since we assume that $\delta>1 /(n-1)$. We inductively define the functions $s^{t}: V^{t} \rightarrow V$. We define $s^{0}=a$ and

$$
s^{t+1}\left(x^{0}, \ldots, x^{t}\right)= \begin{cases}s^{t}\left(x^{0}, \ldots, x^{t-1}\right), & \text { if } x^{t}=s^{t}\left(x^{0}, \ldots, x^{t-1}\right), \\ e_{i^{t+1}\left(x^{t}\right)}, & \text { if } x^{t} \neq s^{t}\left(x^{0}, \ldots, x^{t-1}\right) .\end{cases}
$$

We define the strategy profile $\sigma$ as follows:

1. For each $t \geq 0$, for each history $h=\left(\pi^{0}, x^{0}, r^{0}, \ldots, \pi^{t-1}, x^{t-1}, r^{t-1}, \pi^{t}\right)$, Player $\pi^{t}(1)$ proposes $p^{t}(h)=s^{t}\left(x^{0}, \ldots, x^{t-1}\right)$.

2. After history $\left(\pi^{0}, x^{0}, r^{0}, \ldots, \pi^{t}, x^{t}\right)$, Player $\pi^{t}(k)$, where $k \in\{2, \ldots, n\}$, accepts $x^{t}$ if $\left[x^{t}=s^{t}\left(x^{0}, \ldots, x^{t-1}\right)\right]$ or $\left[i^{t+1}\left(x^{t}\right)=\pi^{t}(\ell)\right.$ and $\left.k>\ell\right]$ and rejects otherwise.

The strategy profile in Theorem 4.2 is similar to the one in Theorem 3.2. The differences are in the calculation of the state of mind and in the acceptance criteria for the players who respond. The state of mind in Theorem 3.2 changes if the proposer demands a payoff greater than the corresponding coordinate of the current state of mind. The state of mind in Theorem 4.2 changes if the proposal is not equal to the current state of mind. In a sense, the state of mind in Theorem 4.2 changes more frequently. In both cases, the state of mind becomes a unit vector after a change. In Theorem 3.2 the state of mind becomes a unit vector that gives one to the responding player with the lowest index for whom a share less than or equal to $1 / 2$ was proposed. In Theorem 4.2 the state of mind becomes a unit vector that gives one to the responding player with the lowest index for whom a share less than $\delta$ was proposed. In Theorem 3.2, the responding players compare a proposal to the state of mind $s^{t+1}\left(x^{0}, \ldots, x^{t}\right)$, which will be proposed next period according to the strategy profile. In Theorem 4.2, the responding players compare a proposal to the state of mind $s^{t}\left(x^{0}, \ldots, x^{t-1}\right)$, which should have been proposed in this period, and also take the order of the responders into account.

The following result is immediate from the definition above.

Claim 4.3 The strategy $\sigma$ has the immediate acceptance property. In particular, according to $\sigma$, Player 1 proposes a in period 0 , which is accepted by Players $2, \ldots, n$.

Claim 4.4 If $1 /(n-1)<\delta<1$, then $\sigma$ is an SPE. 
Proof. We show that at every history none of the players has a profitable one-shot deviation from $\sigma$.

Step 1. Consider a proposer history $\left(\pi^{0}, x^{0}, r^{0}, \ldots, \pi^{t-1}, x^{t-1}, r^{t-1}, \pi^{t}\right)$. The strategy $\sigma$ leads to a payoff of $\delta^{t} s_{\pi^{t}(1)}^{t}\left(x^{0}, \ldots, x^{t-1}\right)$ for Player $\pi^{t}(1)$. Suppose Player $\pi^{t}(1)$ deviates from $\sigma$ and proposes $x^{t} \neq s^{t}\left(x^{0}, \ldots, x^{t-1}\right)$. Since $i^{t+1}\left(x^{t}\right) \neq i^{t}(1)$, the proposal $x^{t}$ is rejected by Player $\pi^{t}(2)$ and next period the proposal $s^{t+1}\left(x^{0}, \ldots, x^{t}\right)=e_{i^{t+1}\left(x^{t}\right)}$ will be made and accepted. This leaves Player $\pi^{t}(1)$ with a payoff of 0 .

STEP 2. Consider a responder history $\left(\pi^{0}, x^{0}, r^{0}, \ldots, \pi^{t}, x^{t}\right)$ and a player $j=\pi^{t}(k)$, where $k \in\{2, \ldots, n\}$.

Consider first the case where $x^{t}=s^{t}\left(x^{0}, \ldots, x^{t-1}\right)$. Strategy $\sigma$ prescribes acceptance and leads to a payoff of $\delta^{t} x_{j}^{t}$ for Player $j$. If $j$ rejects $x^{t}$, next period the proposal $s^{t+1}\left(x^{0}, \ldots, x^{t}\right)=s^{t}\left(x^{0}, \ldots, x^{t-1}\right)=x^{t}$ will be made and accepted, resulting in a payoff of $\delta^{t+1} x_{j}^{t}$ to Player $j$. Hence rejection is not a profitable deviation.

Consider next the case where $x^{t} \neq s^{t}\left(x^{0}, \ldots, x^{t-1}\right)$. It follows that $s^{t+1}\left(x^{0}, \ldots, x^{t}\right)=$ $e_{i^{t+1}\left(x^{t}\right)}$. Let $\ell \in\{1, \ldots, n\}$ be such that $\pi^{t}(\ell)=i^{t+1}\left(x^{t}\right)$. We consider three cases depending on whether $k<\ell, k>\ell$, or $k=\ell$.

Case 1: $k<\ell$. According to $\sigma$, Player $j$ should reject, which leads to a payoff of 0 to Player $j$ since the rejection of $x^{t}$ is followed by the proposal $e_{i^{t+1}\left(x^{t}\right)}$, which is accepted. Suppose Player $j$ accepts $x^{t}$. Since $k+1 \leq \ell$, Player $\pi^{t}(k+1)$ will reject $x^{t}$ according to $\sigma$, so also in this case the proposal $e_{i^{t+1}\left(x^{t}\right)}$ is made and accepted in the next period. We conclude that acceptance is not a profitable deviation.

Case 2: $k>\ell$. According to $\sigma$, Player $j$ accepts, which leads to payoff $\delta^{t} x_{j}^{t}$, because the players following $j$ in the response order all accept $x^{t}$ according to $\sigma$. If $j$ rejects $x^{t}$, next period the proposal $e_{i^{t+1}\left(x^{t}\right)}$ will be made and accepted, leaving Player $j$ with payoff 0 . We conclude that rejection is not a profitable deviation.

Case 3: $k=\ell$. In this case $i^{t+1}\left(x^{t}\right)=j$ and the strategy $\sigma$ prescribes rejection. It leads to a payoff of $\delta^{t+1}$ to Player $j$ since following the rejection of $x^{t}$ the proposal $e_{i^{t+1}\left(x^{t}\right)}$ will be made and accepted next period. Suppose on the other hand that Player $j$ accepts $x^{t}$. Since the players following $j$ in the response order all accept $x^{t}$, this leads to a payoff of $\delta^{t} x_{j}^{t}$. It follows by definition of $i^{t+1}\left(x^{t}\right)$ that $x_{j}^{t} \leq \delta$. We conclude that acceptance is not a profitable deviation.

We have checked that no player has a profitable one-shot deviation from $\sigma$.

Claim 4.5 The strategy profile $\sigma$ has infinite recall. 
Proof. We take $Y=\left\{e_{1}, \ldots, e_{n}, a\right\}$ and, for $t=1, \ldots, n$, we define the function $f^{t}$ : $V \times Y \rightarrow Y$ by

$$
f^{t}(x, y)= \begin{cases}y, & \text { if } x=y \\ e_{i^{t}(x)}, & \text { otherwise }\end{cases}
$$

where $i^{t}(x)=\min \left\{k \neq t \mid x_{k} \leq \delta\right\}$. For $k \in \mathbb{N}$ and $t=1, \ldots, n$, we define $f^{t+n k}=f^{t}$. We verify next that the functions $f^{t}$ thus defined satisfy the conditions of Claim 3.5.

Condition [1] is immediate. To verify Condition [2], we argue as follows. If $a$ is not a member of $\left\{e_{1}, \ldots, e_{n}\right\}$, then $f^{1}\left(e_{1}, a\right)=e_{2} \neq a$. If $a=e_{t}$ for some $t \in\{1, \ldots, n\}$, take any $i \in\{1, \ldots, n\} \backslash\{t\}$ and notice that $f^{t}\left(e_{i}, e_{t}\right)=e_{i^{t}\left(e_{i}\right)} \neq e_{t}$ because $i^{t}\left(e_{i}\right) \neq t$.

To verify condition [3], consider $t \geq 1$ and $y, y^{\prime} \in Y$ with $y \neq y^{\prime}$. We have $f^{t}(y, y)=y$ and $f^{t}\left(y, y^{\prime}\right)=e_{i^{t}(y)}$. Suppose $y=e_{i^{t}(y)}$ and define $j=i^{t}(y)$. Since $i^{t}\left(e_{j}\right) \neq j$, we have that

$$
e_{j}=y=e_{i^{t}}\left(e_{j}\right) \neq e_{j},
$$

a contradiction.

\section{Illustration of the main result}

Consider a three-player bargaining game where only Player 1 can make proposals. Intuition tells us that such a game is hardly of any interest: since Player 1 has all the bargaining power, surely the only outcome that can arise in this game is the one where Player 1 gets the entire surplus? We show this intuition to be wrong. In fact, there exists a subgame perfect equilibrium where Player 2 gets the entire surplus and one where Player 3 does. Furthermore, there is even a subgame perfect equilibrium where the zero vector $(0,0,0)$ is proposed and accepted. Unlike the examples of the preceding sections, the subgame perfect equilibrium strategies constructed below have one-period recall. To compute his proposal in a given period, Player 1 only has to remember his previous proposal and whether it was Player 2 or 3 who rejected it. The responses of Players 2 and 3 only depend on the current proposal.

While these claims are subsumed by our main theorem in the next section, we offer an explicit proof. We do so to illustrate the construction in the next section.

Example 5.1 Let $N=\{1,2,3\}$ and $V=\left\{x \in \mathbb{R}_{+}^{3} \mid x_{1}+x_{2}+x_{3} \leq 1\right\}$. The bargaining game proceeds as described in Section 2, where the period $t$ permutation $\pi^{t}$ is given by $\pi^{t}(i)=i$ for every $t$. So every period Player 1 makes the proposal, after which Player 2 
responds. If Player 2 accepts, it is Player 3's turn to respond to the proposal. The players have a common discount factor $\delta$. We define $U=\left\{e_{0}, e_{2}, e_{3}\right\}$.

Theorem 5.2 Consider the bargaining game of Example 5.1. If $1 / 2<\delta<1$, then for every $a \in U$ there exists a one-period recall SPE such that in period 0 a is proposed by Player 1 and accepted by Players 2 and 3.

Since the permutation $\pi^{t}$ is fixed throughout the game, we omit it from the notation for histories. The strategy profile $\sigma$ is defined as follows.

1. At $t=0$, Player 1 proposes $a$. For each $t \geq 1$, after history $h=\left(x^{0}, r^{0}, \ldots, x^{t-1}, r^{t-1}\right)$, Player 1 proposes

$$
p^{t}(h)=\left\{\begin{array}{lll}
e_{0}, & \text { if } & {\left[x^{t-1} \in U\right],} \\
e_{2}, & \text { if } & {\left[x^{t-1} \notin U \text { and } r^{t-1}=2\right],} \\
e_{3}, & \text { if } & {\left[x^{t-1} \notin U \text { and } r^{t-1}=3\right] .}
\end{array}\right.
$$

2. For $t \geq 0$, after history $\left(x^{0}, r^{0}, \ldots, x^{t-1}, r^{t-1}, x^{t}\right)$, Player 2 accepts $x^{t}$ if $x^{t} \in U$, and rejects otherwise.

3. For $t \geq 0$, after history $\left(x^{0}, r^{0}, \ldots, x^{t-1}, r^{t-1}, x^{t}\right)$, Player 3 accepts $x^{t}$ if $x^{t} \in U$ or $x_{3}^{t} \geq \delta$, and rejects otherwise.

According to the strategy profile, the proposal $a \in U$ is made and accepted in period 0 . Any proposal outside $U$ is rejected by Player 2 , after which proposal $e_{2}$ is made and accepted in the next period.

The intuition behind the strategies is the following. Player 1 is indifferent between any of his actions, because any proposal that Player 2 accepts gives him payoff 0 . Player 2 is encouraged to accept proposals from $U$ and to reject other proposals: after rejection of a proposal that is not from $U, e_{2}$ will be proposed and accepted. The same logic works for Player 3 except that he also accepts proposals that give him payoff higher than or equal to $\delta$.

Rubinstein (1982) has shown that there is a unique subgame perfect equilibrium for the two-player case. Our approach indeed breaks down for the case with two players. Subgame perfection requires the last player who responds to a proposal offering a payoff of at least $\delta$ to accept. In the two-player case, this enables the proposer to obtain at least a payoff of $1-\delta$, and a contraction argument can be used to pin down equilibrium payoffs to a unique value. In cases with three or more players, a proposer cannot make a proposal that guarantees him a strictly positive payoff, as the proposer cannot offer more than $\delta$ to all the responders if $\delta$ is sufficiently high. 
Claim 5.3 The strategy profile $\sigma$ has the immediate acceptance property. If $1 / 2<\delta<1$, the strategy profile $\sigma$ is an SPE.

Proof. The profile $\sigma$ has the immediate acceptance property because Player 1 only makes proposals that belong to $U$ and all members of $U$ are accepted by Players 2 and 3. We verify that the one-shot deviation principle is satisfied.

SteP 1. We show that Player 1 has no profitable one-shot deviations. At each history $h \in H_{1}^{t}$ any of Player 1's actions yields Player 1 a payoff of zero. This follows because the only proposals accepted by Player 2 are $e_{0}, e_{2}$, and $e_{3}$, and each of these proposals gives payoff 0 to Player 1 . Hence Player 1 is indifferent between any of his actions. In particular, Player 1 has no profitable deviations from $\sigma$.

Step 2. We show that Players 2 and 3 have no profitable one-shot deviations. Take a history $h \in H_{2}^{t}$ ending with the proposal $x^{t}$. We consider three cases:

1. $x^{t} \in U$,

2. $x^{t} \notin U$ and $x_{3}^{t}<\delta$,

3. $x^{t} \notin U$ and $x_{3}^{t} \geq \delta$.

Case 1: $x^{t} \in U$.

The strategy $\sigma$ calls for the acceptance of $x^{t}$. If Player 2 or Player 3 deviates and rejects, the proposal $e_{0}$ is made and accepted next period. Clearly rejection is not a profitable deviation for the responding players.

Case 2: $x^{t} \notin U$ and $x_{3}^{t}<\delta$.

Consider Player 3. The strategy $\sigma$ recommends that Player 3 rejects. Rejection gives Player 3 a payoff of $\delta^{t+1}$, because the proposal $e_{3}$ is made and accepted in period $t+1$. If Player 3 deviates and accepts the proposal, he obtains payoff $\delta^{t} x_{3}^{t}<\delta^{t+1}$. So acceptance is not a profitable deviation.

Consider Player 2. The strategy $\sigma$ calls for Player 2 to reject. Rejection gives Player 2 a payoff of $\delta^{t+1}$, because the proposal $e_{2}$ is made and accepted next period. Suppose now Player 2 deviates and accepts $x^{t}$. Then Player 3 rejects $x^{t}$ and in period $t+1$ the proposal $e_{3}$ is made and accepted, leaving Player 2 with payoff zero. So acceptance is not a profitable deviation.

Case 3: $x^{t} \notin U$ and $x_{3}^{t} \geq \delta$.

Consider Player 3. According to $\sigma$ Player 3 should accept. Acceptance gives Player 3 the payoff $\delta^{t} x_{3}^{t} \geq \delta^{t+1}$. If Player 3 deviates and rejects the proposal, the proposal $e_{3}$ is made and accepted in period $t+1$, yielding Player 3 the payoff $\delta^{t+1}$. So rejection is not a profitable deviation. 
Consider Player 2. According to the strategy $\sigma$ Player 2 must reject. Rejection gives Player 2 a payoff of $\delta^{t+1}$, because the proposal $e_{2}$ is made and accepted in period $t+1$. Suppose now Player 2 deviates and accepts. Since Player 3 accepts $x^{t}$, Player 2 then receives a payoff of $\delta^{t} x_{2}^{t}$. Now $x_{2}^{t} \leq 1-x_{3}^{t} \leq 1-\delta \leq \delta$, so $\delta^{t} x_{2}^{t} \leq \delta^{t+1}$. So acceptance is not a profitable deviation.

Our main result generalizes the example above in a number of ways. As has been discussed in Section 2, we allow nature's choice of the permutations to be probabilistic and history dependent, where the choice of nature may even depend on aspects of the history of more than one period ago. Secondly, we only impose rather minimal assumptions on the set $V$ of feasible payoffs. And finally, we show that any vector in the set $V$, not just the unit vectors, can be supported as a subgame perfect equilibrium outcome.

\section{The folk theorem for bargaining games}

In this section we prove our main result: if the players are sufficiently patient, given any point $a$ of the set $V$ one can find a subgame perfect equilibrium having one-period recall such that on the equilibrium path of play the point $a$ is proposed and accepted in period zero. As a first step we identify the level of patience needed for the result to hold.

Lemma 6.1 There exists $\bar{\delta}<1$ such that for every $v \in V$ the set $\left\{i \in N: v_{i} \geq \bar{\delta}\right\}$ contains at most one point.

Proof. Suppose not. Then there exists $i, j \in N$ with $i \neq j$ and a sequence $\left(v^{m}\right)_{m \in \mathbb{N}}$ of points in $V$ such that $v_{i}^{m} \geq 1-1 / m$ and $v_{j}^{m} \geq 1-1 / m$. Since the set $V$ is compact, we can assume without loss of generality that $v^{m}$ converges to $\bar{v} \in V$. But then $\bar{v}_{i} \geq 1$ and $\bar{v}_{j} \geq 1$, contradicting Assumption 4 .

For the rest of this section we fix some $\bar{\delta}<1$ such that Lemma 6.1 is satisfied. We are now in a position to state our main result.

Theorem 6.2 Given any point $a \in V$ there exists a strategy profile $\sigma$ such that:

[1] The proposal a is made in period 0 and is unanimously accepted. Furthermore, the strategy $\sigma$ has the immediate acceptance property.

[2] The strategy $\sigma$ has $1-$ period recall.

[3] If for every $i \in N$ it holds that $\delta_{i} \geq \bar{\delta}$, then $\sigma$ is an SPE. 
We define our strategy profile by specifying actions for a player who is in a particular position in the game. These actions do not depend on the identity of the player. There are $n$ positions in the game: the proposer, the player who responds first, the player who responds second, and so on. The strategy for the proposer, $\pi^{t}(1)$, specifies the proposal at each period $t$, as a function $p^{t}(h)$ of the history of play up to $t$. The strategies for "the player who responds first", Player $\pi^{t}(2)$, "the player who responds second", Player $\pi^{t}(3), \ldots$, and "the player who responds last", Player $\pi^{t}(n)$, specifies their reaction to the proposal contingent on the history of play up to $t$.

We define $E=\left\{e_{0}\right\} \cup\left\{e_{i} \mid i \in N\right\}$. We define the strategy $\sigma$ as follows:

1. In period $t=0$, Player $\pi^{0}(1)$ proposes $p^{0}=a$. At $t=1$, after history $h=$ $\left(\pi^{0}, x^{0}, r^{0}, \pi^{1}\right)$, Player $\pi^{1}(1)$ proposes

$$
p^{1}(h)= \begin{cases}e_{0}, & \text { if } \quad x^{0}=a \\ e_{r^{0}}, & \text { if } \quad x^{0} \neq a .\end{cases}
$$

For each $t \geq 2$, after history $h=\left(\pi^{0}, x^{0}, r^{0}, \ldots, \pi^{t-1}, x^{t-1}, r^{t-1}, \pi^{t}\right)$, Player $\pi^{t}(1)$ proposes

$$
p^{t}(h)= \begin{cases}e_{0}, & \text { if } \quad x^{t-1} \in E \\ e_{r^{t-1}}, & \text { if } \quad x^{t-1} \notin E .\end{cases}
$$

2. After history $\left(\pi^{0}, x^{0}, r^{0}, \ldots, \pi^{t-1}, x^{t-1}, r^{t-1}, \pi^{t}, x^{t}\right)$, Player $\pi^{t}(j), j \in\{2, \ldots, n-1\}$, accepts $x^{t}$ if

- $\left[x^{t}=p^{t}\left(\pi^{0}, x^{0}, r^{0}, \ldots, \pi^{t}\right)\right]$ or

- $\left[t \geq 1\right.$ and $x^{t} \in E$ and $\left.x^{t} \neq e_{\pi^{t}(1)}\right]$,

and rejects otherwise.

3. After history $\left(\pi^{0}, x^{0}, r^{0}, \ldots, \pi^{t-1}, x^{t-1}, r^{t-1}, \pi^{t}, x^{t}\right)$, Player $\pi^{t}(n)$ accepts $x^{t}$ if

- $\left[x^{t}=p^{t}\left(\pi^{0}, x^{0}, r^{0}, \ldots, \pi^{t}\right)\right]$ or

- $\left[t \geq 1\right.$ and $x^{t} \in E$ and $\left.x^{t} \neq e_{\pi^{t}(1)}\right]$ or

- $\left[x_{\pi^{t}(n)}^{t} \geq \delta_{\pi^{t}(n)}\right]$,

and rejects otherwise. 
Part [1] of Theorem 6.2 is immediate from the definition above: indeed, for every $h \in H_{1}^{t}$ the proposal $p^{t}(h)$ is accepted by all the responders. In particular, the period 0 proposal $p^{0}=a$ is unanimously accepted.

Part [2] is equally straightforward: Notice that every proposer history in period $t \geq 1$ is of the form $\left(h, x^{t-1}, r^{t-1}, \pi^{t}\right)$ where $h \in H_{1}^{t-1}$ is some proposer history in period $t-1$, while a responder history at $t$ can be written as $\left(h, x^{t-1}, r^{t-1}, \pi^{t}, x^{t}\right)$ where $h \in H_{1}^{t-1}$. It is clear from the definition above that the proposal $p^{t}\left(h, x^{t-1}, r^{t-1}, \pi^{t}\right)$ at the proposal history $\left(h, x^{t-1}, r^{t-1}, \pi^{t}\right)$ does not depend on $h$. And hence also the response $\sigma_{i}\left(h, x^{t-1}, r^{t-1}, \pi^{t}, x^{t}\right)$ of Player $i \neq \pi^{t}(1)$ at the responder history $\left(h, x^{t-1}, r^{t-1}, \pi^{t}, x^{t}\right)$ does not depend on $h$. It follows that the strategy profile $\sigma$ has one-period recall.

We proceed to prove part [3] of the theorem. The intuition behind our construction could be summarized as follows. According to $\sigma$ the responders only accept a proposal $p^{t}(h)$ as prescribed by the strategy $\sigma$, along with those unit vectors which give 0 to the proposer. This implies that any deviation by a proposer can only result in a payoff of zero, the essence of Claim 6.3 below. Rejections of proposals in the set $E$ are penalized by having the next proposer offer the zero vector $e_{0}$. Rejections of proposals outside the set $E$ are rewarded by having the next proposer offer the maximum payoff of 1 to the rejector.

The proof of part [3] of Theorem 6.2 is divided into three steps. Claim 6.3 shows that for each proposer history $h \in H_{1}^{t}$ any proposal other than $p^{t}(h)$ yields the proposer a payoff of zero. This implies in particular that no proposer has a profitable one-shot deviation from $\sigma$. Claim 6.4 shows that there are no profitable one-shot deviations from $\sigma$ at the responder histories in period 0, and Claim 6.5 that there are no such deviations at period $t \geq 1$. In Claims 6.4 and 6.5 we assume that for every $i \in N, \delta_{i} \geq \bar{\delta}$.

Claim 6.3 Consider a proposer history $h \in H_{1}^{t}$. If a proposal $x^{t} \neq p^{t}(h)$ is made after history $h$, it leads to a payoff of 0 for the proposer. In particular, there are no profitable one-shot deviations from $\sigma$ at $h$.

Proof. Suppose the history $h$ ends with the permutation $\pi^{t}$. Take any $x^{t} \neq p^{t}(h)$. We consider two cases depending on whether $t=0$ or $t \geq 1$.

- Case $t=0$. We have $p^{0}=a$. If a proposal $x^{0} \neq a$ is made, it is rejected by Player $\pi^{0}(2)$, and in period 1 the proposal $e_{\pi^{0}(2)}$ will be made and accepted, see part [1] of Theorem 6.2. This leads to payoff 0 for Player $\pi^{0}(1)$.

- Case $t \geq 1$. We consider three subcases:

1. Case $x^{t}=e_{\pi^{t}(1)}$. Since $x^{t} \neq p^{t}(h)$, the proposal $x^{t}$ is rejected by Player $\pi^{t}(2)$. Since the period $t+1$ proposal $e_{0}$ is accepted by part [1] of Theorem 6.2, Player $\pi^{t}(1)$ receives 0. 
2. Case $x^{t} \in E \backslash\left\{e_{\pi^{t}(1)}\right\}$. In this case the proposal $x^{t}$ is unanimously accepted. Player $\pi^{t}(1)$ receives payoff 0 .

3. Case $x^{t} \notin E$. In this case the proposal $x^{t}$ is rejected by Player $\pi^{t}(2)$. The next proposal is $e_{\pi^{t}(2)}$, which is accepted. This leads to payoff 0 for Player $\pi^{t}(1)$.

In each case Player $\pi^{t}(1)$ receives a payoff of zero.

Claim 6.4 Consider a responder history $h \in H_{2}^{0}$. If, for every $i \in N, \delta_{i} \geq \bar{\delta}$, then there are no profitable one-shot deviations from $\sigma$ at $h$.

Proof. Let $h=\left(\pi^{0}, x^{0}\right)$. We consider the following three cases:

1. $x^{0}=a$,

2. $x^{0} \neq a$ and $x_{\pi^{0}(n)}^{0} \geq \delta_{\pi^{0}(n)}$,

3. $x^{0} \neq a$ and $x_{\pi^{0}(n)}^{0}<\delta_{\pi^{0}(n)}$.

Case 1: $x^{0}=a$. Consider Player $\pi^{0}(j)$ where $j \in\{2, \ldots, n\}$. Strategy $\sigma$ prescribes that the proposal $x^{0}$ be accepted. Suppose Player $\pi^{0}(j)$ rejects $x^{0}$ instead. Then in period 1 the proposal $e_{0}$ will be made and accepted. Since 0 is the lowest payoff in the game, rejection is not a profitable deviation.

Case 2: $x^{0} \neq a$ and $x_{\pi^{0}(n)}^{0} \geq \delta_{\pi^{0}(n)}$.

Consider Player $\pi^{0}(n)$. According to $\sigma$ Player $\pi^{0}(n)$ accepts $x^{0}$ and receives a payoff of $x_{\pi^{0}(n)}^{0} \geq \delta_{\pi^{0}(n)}$. If Player $\pi^{0}(n)$ deviates and rejects, in period 1 the proposal $e_{\pi^{0}(n)}$ is made and accepted. It leads to a payoff of $\delta_{\pi^{0}(n)}$ for Player $\pi^{0}(n)$, so the deviation is not profitable.

Consider Player $\pi^{0}(n-1)$. According to $\sigma$ Player $\pi^{0}(n-1)$ rejects $x^{0}$, the proposal $e_{\pi^{0}(n-1)}$ is made and accepted next period, and Player $\pi^{0}(n-1)$ receives payoff $\delta_{\pi^{0}(n-1)}$. Suppose now Player $\pi^{0}(n-1)$ deviates and accepts $x^{0}$. Since according to $\sigma$ Player $\pi^{0}(n)$ accepts $x^{0}$, Player $\pi^{0}(n-1)$ receives the payoff $x_{\pi^{0}(n-1)}^{0}$. Now since $x^{0}$ belongs to $V$ and $x_{\pi^{0}(n)}^{0} \geq \delta_{\pi^{0}(n)} \geq \bar{\delta}$, we have $x_{\pi^{0}(n-1)}^{0}<\bar{\delta} \leq \delta_{\pi^{0}(n-1)}$ by the choice of $\bar{\delta}$. So the deviation is not profitable.

Consider Player $\pi^{0}(j)$ where $j \in\{2, \ldots, n-2\}$. According to the strategy profile $\sigma$ Player $\pi^{0}(j)$ rejects, and the proposal $e_{\pi^{0}(j)}$ is made and accepted next period. This leads to a payoff of $\delta_{\pi^{0}(j)}$. Suppose now Player $\pi^{0}(j)$ deviates and accepts $x^{0}$. Then $x^{0}$ will be rejected by Player $\pi^{0}(j+1)$, and the next proposal $e_{\pi^{0}(j+1)}$ will be made and accepted. This leads to a payoff of zero to Player $\pi^{0}(j)$. So the deviation is not profitable.

Case 3: $x^{0} \neq a$ and $x_{\pi^{0}(n)}^{0}<\delta_{\pi^{0}(n)}$. 
Consider Player $\pi^{0}(n)$. According to $\sigma$ Player $\pi^{0}(n)$ rejects, and the proposal $e_{\pi^{0}(n)}$ is made and accepted in period 1, leading to a payoff of $\delta_{\pi^{0}(n)}$ to Player $\pi^{0}(n)$. If Player $\pi^{0}(n)$ deviates and accepts, he receives only $x_{\pi^{0}(n)}^{0}<\delta_{\pi^{0}(n)}$, so the deviation is not profitable.

Consider Player $\pi^{0}(j)$ where $j \in\{2, \ldots, n-1\}$. According to $\sigma$ Player $\pi^{0}(j)$ rejects, and the proposal $e_{\pi^{0}(j)}$ is made and accepted in period 1, leading to a payoff of $\delta_{\pi^{0}(j)}$ for Player $\pi^{0}(j)$. Suppose Player $\pi^{0}(j)$ deviates and accepts. Then $x^{0}$ will be rejected by Player $\pi^{0}(j+1)$ and next period the proposal $e_{\pi^{0}(j+1)}$ will be made and accepted, thus leaving Player $\pi^{0}(j)$ with zero payoff. Thus the deviation is not profitable.

Claim 6.5 Consider a responder history $h \in H_{2}^{t}$ where $t \geq 1$. If, for every $i \in N, \delta_{i} \geq \bar{\delta}$, then there are no profitable one-shot deviations from $\sigma$ at $h$.

Proof. The history $h$ is of the form $h=\left(h^{\prime}, \pi^{t}, x^{t}\right)$ where $h^{\prime} \in H_{3}^{t-1}, \pi^{t}$ is the permutation chosen by nature at $h^{\prime}$, and $x^{t}$ is the proposal chosen by the Player $\pi^{t}(1)$ at $\left(h^{\prime}, \pi^{t}\right)$. We consider the following cases:

1. $x^{t} \in E \backslash\left\{e_{\pi^{t}(1)}\right\}$,

2. $x^{t}=e_{\pi^{t}(1)}$,

3. $x^{t} \notin E$ and $x_{\pi^{t}(n)}^{t} \geq \delta_{\pi^{t}(n)}$,

4. $x^{t} \notin E$ and $x_{\pi^{t}(n)}^{t}<\delta_{\pi^{t}(n)}$.

Case 1: $x^{t} \in E \backslash\left\{e_{\pi^{t}(1)}\right\}$.

According to the strategy $\sigma$ all the responders $\pi^{t}(j)$ for $j \in\{2, \ldots n\}$ accept $x^{t}$. In case of a rejection, next period the proposal $e_{0}$ is made and accepted. Since 0 is the lowest possible payoff in the game, a deviation is not profitable.

Case 2: $x^{t}=e_{\pi^{t}(1)}$.

Either action of Player $\pi^{t}(j)$ for $j \in\{2, \ldots, n\}$ results in a payoff of zero. Indeed, if the proposal $x^{t}$ is accepted, Player $\pi^{t}(j)$ receives $x_{\pi^{t}(j)}^{t}=0$. And if $x^{t}$ is rejected, the next proposal will be $e_{0}$, which is accepted. In either case Player $\pi^{t}(j)$ receives payoff 0 . It follows that there are no profitable one-shot deviations from $\sigma$ at $h$.

Case 3: $x^{t} \notin E$ and $x_{\pi^{t}(n)}^{t} \geq \delta_{\pi^{t}(n)}$.

Notice that $x^{t} \neq p^{t}\left(h^{\prime}, \pi^{t}\right)$ because for $t \geq 1$ the proposal $p^{t}\left(h^{\prime}, \pi^{t}\right)$ belongs to $E$.

Consider Player $\pi^{t}(n)$. According to $\sigma$ Player $\pi^{t}(n)$ accepts $x^{t}$ and receives a payoff of $\delta_{\pi^{t}(n)}^{t} x_{\pi^{t}(n)}^{t} \geq \delta_{\pi^{t}(n)}^{t+1}$. If Player $\pi^{t}(n)$ deviates and rejects, in period $t+1$ the proposal $e_{\pi^{t}(n)}$ is made and accepted. This leads to a payoff of $\delta_{\pi^{t}(n)}^{t+1}$ for Player $\pi^{t}(n)$, so the deviation is not profitable. 
Consider Player $\pi^{t}(n-1)$. According to $\sigma$ Player $\pi^{t}(n-1)$ rejects $x^{t}$, the proposal $e_{\pi^{t}(n-1)}$ is made and accepted next period, and Player $\pi^{t}(n-1)$ receives payoff $\delta_{\pi^{t}(n-1)}^{t+1}$. Suppose now Player $\pi^{t}(n-1)$ deviates and accepts $x^{t}$. Since according to $\sigma$ Player $\pi^{t}(n)$ accepts $x^{t}$, Player $\pi^{t}(n-1)$ then receives the payoff $\delta_{\pi^{t}(n-1)}^{t} x_{\pi^{t}(n-1)}^{t}$. Now since $x^{t}$ is a point of $V$ and $x_{\pi^{t}(n)}^{t} \geq \delta_{\pi^{t}(n)} \geq \bar{\delta}$, we have $x_{\pi^{t}(n-1)}^{t}<\bar{\delta} \leq \delta_{\pi^{t}(n-1)}$, by the choice of $\bar{\delta}$. Hence $\delta_{\pi^{t}(n-1)}^{t} x_{\pi^{t}(n-1)}^{t}<\delta_{\pi^{t}(n-1)}^{t+1}$ and the deviation is not profitable.

Consider Player $\pi^{t}(j)$ where $j \in\{2, \ldots, n-2\}$. According to the strategy profile $\sigma$ Player $\pi^{t}(j)$ rejects, and the proposal $e_{\pi^{t}(j)}$ is made and accepted next period. This leads to a payoff of $\delta_{\pi^{t}(j)}^{t+1}$. Suppose now Player $\pi^{t}(j)$ deviates and accepts $x^{t}$. Then $x^{t}$ will be rejected by Player $\pi^{t}(j+1)$, and the next period proposal $e_{\pi^{t}(j+1)}$ will be made and accepted. This leads to a payoff of zero to Player $\pi^{t}(j)$. So the deviation is not profitable.

Case 4: $x^{t} \notin E$ and $x_{\pi^{t}(n)}^{t}<\delta_{\pi^{t}(n)}$.

Notice that $x^{t}$ is not equal to $p^{t}\left(h^{\prime}, \pi^{t}\right)$ since for $t \geq 1$ the latter is an element of $E$.

Consider Player $\pi^{t}(n)$. According to $\sigma$ Player $\pi^{t}(n)$ rejects, and the proposal $e_{\pi^{t}(n)}$ is made and accepted in period $t+1$, leading to a payoff of $\delta_{\pi^{t}(n)}^{t+1}$ to Player $\pi^{t}(n)$. If Player $\pi^{t}(n)$ deviates and accepts, he receives only a payoff of $\delta_{\pi^{t}(n)}^{t} x_{\pi^{t}(n)}^{t}<\delta_{\pi^{t}(n)}^{t+1}$, so the deviation is not profitable.

Consider Player $\pi^{t}(j)$ where $j \in\{2, \ldots, n-1\}$. According to $\sigma$ Player $\pi^{t}(j)$ rejects, and the proposal $e_{\pi^{t}(j)}$ is made and accepted in period $t+1$, leading to a payoff of $\delta_{\pi^{t}(j)}^{t+1}$ for Player $\pi^{t}(j)$. Suppose Player $\pi^{t}(j)$ deviates and accepts. Then $x^{t}$ will be rejected by Player $\pi^{t}(j+1)$ and the proposal $e_{\pi^{t}(j+1)}$ will be made and accepted in period $t+1$, thus leaving Player $\pi^{t}(j)$ with zero payoff. Thus the deviation is not profitable.

\section{Conclusion}

In this paper we have proved a folk theorem for a general class of bargaining games with three or more players. We show that for any vector of feasible payoffs there is a subgame perfect equilibrium such that the vector of feasible payoffs is proposed and accepted in period 0 . The subgame perfect equilibrium strategies are pure and have one-period recall. The existing literature has shown such a result only for specific examples of bargaining games and relied on strategies having infinite recall.

Our bargaining protocol is sufficiently general to cover many bargaining protocols studied in the literature as special cases. Moreover, it covers cases that have not been studied in the literature so far, where for instance the protocol depends on proposals that have been made in previous rounds. Although our equilibrium strategies have one-period recall, the protocol itself is allowed to depend on aspects of the history that are related to arbitrarily 
many periods before. Several authors have found that the assumption of bounded recall may severely limit the set of outcomes that can occur in an equilibrium. On the other hand, Hörner and Olszewski (2009) and Mailath and Olszewski (2011) provide folk theorems for repeated games where strategies have bounded recall. Our results show that not even one-period recall is sufficient to limit the set of subgame perfect equilibrium payoffs in multilateral bargaining games.

\section{References}

Aumann, R.J. (1981), "Survey of Repeated Games," in V. Böhm and H.H. Nachtkamp (eds.), Essays in Game Theory and Mathematical Economics in Honor of Oskar Morgenstern, Bibliografisches Institut Mannheim, pp. 11-42.

Banks, J., And J. Duggan (2000), "A Bargaining Model of Collective Choice," American Political Science Review, 94, 73-88.

Bhaskar, V., G.J. Mailath, and S. Morris (2009), "A Foundation for Markov Equilibria in Infinite Horizon Perfect Information Games," PIER Working Paper, 09-029, $1-21$.

Binmore, K. (1987), "Perfect Equilibria in Bargaining Models," in K. Binmore and P. Dasgupta (eds.), The Economics of Bargaining, Basil Blackwell, Oxford, UK, pp. $77-105$.

Binmore, K., M.J. Osborne, And A. Rubinstein (1992), "Non-Cooperative Models of Bargaining," in R.J. Aumann and S. Hart (eds.), Handbook of Game Theory, Volume 1, pp. 179-225.

Chatterjee, K., B. Dutta, D. Ray, and K. Sengupta (1993), "A Noncooperative Theory of Coalitional Bargaining," Review of Economic Studies, 60, 463-477.

Chen, B. (2008), "On Effective Minimax Payoffs and Unequal Discounting," Economics Letters, 100, 105-107.

Cole, H.L., and N. Kocherlakota (2005), "Finite Memory and Imperfect Monitoring," Games and Economic Behavior, 53, 59-72.

Dutta, P.K. (1995), "A Folk Theorem for Stochastic Games," Journal of Economic Theory, 66, 1-32. 
Friedman, J. (1971), "A Non-cooperative Equilibrium for Supergames," Review of Economic Studies, 38, 1-12.

FudenberG, D., And E. Maskin (1986), "The Folk Theorem in Repeated Games with Discounting or with Incomplete Information," Econometrica, 54, 533-554.

Fudenberg, D., And J. Tirole (1991), Game Theory, MiT Press, Cambridge, Massachusetts.

FudenberG, D., And Y. Yamamoto (2011), "The Folk Theorem For Irreducible Stochastic Games with Imperfect Public Monitoring," Journal of Economic Theory 146, 1664-1683.

HALleR, H. (1986), "Non-cooperative Bargaining of $N \geq 3$ Players," Economics Letters, 22, 11-13.

Harsanyi, J.C. (1973), "Games with Randomly Disturbed Payoffs: A New Rationale for Mixed-strategy Equilibrium Points," International Journal of Game Theory, 2, $1-23$.

Herings, P.J.J., And A. Predtetchinski (2010), "One-dimensional Bargaining with Markov Recognition Probabilities," Journal of Economic Theory, 145, 189-215.

Herrero, M.J. (1985), A Strategic Bargaining Approach to Market Institutions, PhD Thesis, London School of Economics, 1-111.

Hörner, J., AND W. Olszewski (2009), "How Robust Is the Folk Theorem," Quarterly Journal of Economics, 124, 1773-1814.

Kalandrakis, T. (2006), "Regularity of Pure Strategy Equilibrium Points in a Class of Bargaining Games," Economic Theory, 28, 309-329.

Lehrer, E., And A. Pauzner (1999), "Repeated Games with Differential Time Preferences," Econometrica, 67, 393-412.

Mailath, G.J., And Olszewski (2011), "Folk Theorems with Bounded Recall under (Almost) Perfect Monitoring," Games and Economic Behavior, 71, 174-192.

Merlo, A., And C. Wilson (1995), "A Stochastic Model of Sequential Bargaining with Complete Information," Econometrica, 63, 371-399.

Rubinstein, A., (1979) "Equilibrium in Supergames with the Overtaking Criterion," Journal of Economic Theory, 21, 1-9. 
Rubinstein, A. (1982), "Perfect Equilibrium in a Bargaining Model," Econometrica, 50, 97-109.

Sabourian, H. (1998), "Repeated Games with $M$-Period Bounded Memory (Pure Strategies)," Journal of Mathematical Economics, 30, 1-35.

Selten, R. (1981), "A Noncooperative Model of Characteristic Function Bargaining," in V. Böhm and H.H. Nachtkamp (eds.), Essays in Game Theory and Mathematical Economics in Honor of Oskar Morgenstern, Bibliografisches Institut Mannheim, pp. $131-151$. 